\title{
Transport and current reversal in stochastically driven ratchets
}

\author{
Mark M. Millonas \\ Complex Systems Group and Center for Nonlinear Studies, Theoretical Division, \\ MS B258 Los Alamos National Laboratory, Los Alamos, NM 87545 \\ \& Santa Fe Institute, Santa Fe, NM 87501 \\ Mark I. Dykman \\ Department of Physics, Stanford University, Stanford, CA 94306.
}

July 27, 2021

\begin{abstract}
We present analytic results for the current in a system moving in an arbitrary periodic potential and driven by weak Gaussian noise with an arbitrary power spectrum which are valid to order $\left(t_{c} / t_{r}\right)^{2}$, where $t_{c}$ is the largest characteristic time of the noise, and $t_{r}$ is the characteristic intrawell relaxation time. The dependence of the current on the shape of the potential, and on the shape of the power spectrum of the noise is illustrated. It is demonstrated that the direction of the current is opposite when the power spectrum of the noise has minimum or maximum at zero frequency. A simple physical mechanism for this behavior is suggested. The behavior of the system in the limit of slow noise $\left(t_{c}>>t_{r}\right)$ is also discussed.
\end{abstract}

Recently some intriguing work has appeared on the subject of stochastically driven ratchets, [1, 2] and the transformation of noise in spatiallyperiodic (or phase-periodic) systems into a current. Such systems are noise rectifiers and thus are of great interest in relation to the understanding of machinery which operates in "the Brownian regime" [3] that is, on the very 
small scale where fluctuations play a major role, and where the basic macroscopic methods of controlling energy flow no longer remain valid. The most immediate examples are biological systems, [4] but these ideas may not be irrelevant in the area of applied technology, where a great interest has developed recently in the possible construction of nanoscale devices. [5]

A simple model for a ratchet is a noise-driven overdamped nonlinear dynamical system described by the stochastic differential equation

$$
\dot{x}=-U^{\prime}(x)+f(t), \quad U(x)=U(x+\lambda), \quad\langle f(t)\rangle=0,
$$

where $U(x)$ is a periodic potential, such as the one illustrated in Fig. 1, and $f(t)$ is zero-mean noise of some type. The theoretical problem is to find the stationary current density $j=\langle\dot{x}(t)\rangle$ in the ratchet given the shape of $U(x)$ and the properties of the noise $f(t)$, and then to find the most appropriate conditions for the transformation of the noise into the current. If the noise is white then the system is (quasi)thermal, the second law of thermodynamics applies, and $j=0$. If the noise is not white, i.e., for colored noise, the system is no longer in thermal equilibrium, and in general $j \neq 0$. Since onset of a current means breaking the "right-left" symmetry, currents may only arise, in the case of additive noise, if the potential $U(x)$ is asymmetric with respect to its extrema. The onset of a current can be viewed also as an example of "temporal order coming out of disorder", since the current is apparently time-irreversible, whereas stationary noise does not distinguish "future" from the "past"; we notice, however, that Eq. (1) implies relaxation and is thus time-irreversible itself.

We consider the case where $f(t)$ is zero-mean Gaussian noise with a frequency-dependent power spectrum

$$
\Phi(\omega)=\int_{-\infty}^{\infty} d t \exp (i \omega t) \phi(t), \quad \phi(t)=<f(t) f(0)>
$$

If the characteristic noise intensity $D=\max \Phi(\omega)$ is small then most of the time the system performs small-amplitude fluctuations about the minima of the potential. Occasionally it "jumps" from the minimum it occupied to the one on the right or left, with the probabilities per unit time $W_{+}$and $W_{-}$, respectively. These jumps give rise to the current $j=\lambda\left(W_{+}-W_{-}\right)$. The dependence of the transition probabilities $W_{ \pm}$on the noise intensity $D$ is of the activation type for Gaussian noise, $W_{ \pm}=$const. $\times \exp \left(-R_{ \pm} / D\right)$. The 
characteristic activation energies $R_{ \pm}$of the escapes from a given minimum $x_{0}$ of the potential over the right $\left(x_{+}\right)$or left $\left(x_{-}\right)$maximum are given by the solution of a variational problem formulated in [6]. In the case where the bandwidth of the spectrum $\Phi(\omega)$ greatly exceeds the reciprocal relaxation time of the system $t_{r}^{-1}=U^{\prime \prime}\left(x_{0}\right)$ the activation energies are $R_{ \pm}=2 F(0) \Delta U+$ $\gamma_{ \pm} F^{\prime \prime}(0)$, where

$$
\begin{gathered}
\Delta U=U(b)-U(a)>0, \quad \gamma_{ \pm}=\int_{x_{0}}^{x_{ \pm}} d x U^{\prime}(x)\left[U^{\prime \prime}(x)\right]^{2} \geq 0, \\
F(\omega)=D / \Phi(\omega), \quad F^{\prime \prime}(\omega)=\frac{d^{2} F(\omega)}{d \omega^{2}}, \quad\left|F^{\prime \prime}(0) / F(0)\right|<<t_{r}^{2} .
\end{gathered}
$$

The current is then given by

$$
j=\lambda W_{K}\left[\exp \left(-\gamma_{+} F^{\prime \prime}(0) / D\right)-\exp \left(-\gamma_{-} F^{\prime \prime}(0) / D\right)\right],
$$

where $W_{K}$ is the Kramer's activation rate

$$
W_{K}=\frac{\pi D / F(0)}{\sqrt{U^{\prime \prime}\left(x_{0}\right)\left|U^{\prime \prime}\left(x_{+}\right)\right|}} \exp (-2 F(0) \Delta U / D)
$$

Eq. 5 is a principle result of this letter. It is immediately obvious from (5) that: (i) the noise color does give rise to the onset of a current due to the fluctuational interwell transitions, and (ii) the direction of the current depends crucially on the shape of the spectral density since $F^{\prime \prime}(0)$ can take both positive or negative signs. We emphasize that, although the corrections $\gamma_{ \pm} F^{\prime \prime}(0)$ to $R_{ \pm}$are small compared to the main term, they are not small compared to the noise intensity $D$ and can change $W_{ \pm}$by orders of magnitude (we have neglected the corrections to the prefactor in $W_{ \pm}$due to the noise color and used the standard Kramers expression for this prefactor valid for white-noise driven systems). In fact, except the special case where $U(x)$ is symmetric with respect to $x_{0}$, the ratio of the probabilities $W_{+} / W_{-}$is exponentially large or small for small intensity of the colored noise, and therefore the transitions in one direction dominate overwhelmingly over the transitions in the opposite direction, so that $|j| \approx \lambda W_{>}$where $W_{>}=\max \left(W_{+}, W_{-}\right)$. The direction of the current is determined by the interplay of the shape of the potential and the features (the shape of the power spectrum, in the present case) of the noise. 
The dependence of the current on the noise color is different depending on the sign of $F^{\prime \prime}(0)$. When $F^{\prime \prime}(0)>0$ there is a saturation effect where, given the noise strength $D$ and the shape of the potential, the current is maximal for $F^{\prime \prime}(0)$ given by

$$
F_{m}^{\prime \prime}(0)=\frac{D \ln \left(\gamma_{-} / \gamma_{+}\right)}{\left(\gamma_{-}-\gamma_{+}\right)}, \quad F_{m}^{\prime \prime}(0)>0 .
$$

Thus, for $F^{\prime \prime}(0)>0, F_{m}^{\prime \prime}(0)$ is the optimal noise color for a given noise strength and ratchet potential.

Eq.(5) also provides an answer to the following question: given the barrier height $\Delta U$, what is the shape of the well for which the current (5) will be most pronounced for weak noise color, that is, what is the most effective shape of the ratchet? This shape is given by the extreme value of $\gamma_{ \pm}$for given $\Delta U$ and period $\lambda$. It is straightforward to show that the corresponding variational problem does not have a differentiable solution: the single-valued potential has to be of the shape approaching the one pictured in Fig. 2. A simple analysis can be done, e.g., for $U^{\prime}(x)=A_{1}+A_{2} \tanh \left[\left(x-\tilde{x}_{0}\right) / a\right],-\lambda / 2<$ $x<\lambda / 2\left(A_{1}+A_{2} \tanh \left[\left(x_{0}-\tilde{x}_{0}\right) / a\right]=0\right)$ : for small $a / \lambda$ the shape of the corresponding potential is close to that of the sawtooth.

The above results apply immediately to the most often studied case where $f(t)$ is exponentially correlated,

$$
\phi(t)=\frac{D}{2 \tau} \exp (-|t| / \tau), \quad \Phi(\omega)=D /\left(1+\omega^{2} \tau^{2}\right) .
$$

The problem of fluctuational transitions induced by this noise has been investigated for small noise intensities in very much detail (see [7], [8], [9] and also [10 for a review). The noise (8) has one correlation time, $\tau$, and $F^{\prime \prime}(0)=2 \tau^{2}>0$. Obviously, $j$ vanishes to first order in $\tau$, in agreement with the results of [2]. It has a maximum as a function of $\tau$ for $\tau$ given by (7), and falls down for large $\gamma_{ \pm} \tau^{2} / D$. The saturation of $j$ vs. $\tau$ is illustrated in Fig. 3.

Naturally occurring noise will generally not be exponentially correlated, a situation which any realistic physical theory must accommodate. This is clearly the regime in which $j \neq 0$. The advantage of the result presented here is that it not only gives an analytic result to second order in $t_{c} / t_{r}$, but is valid for any Gaussian noise. A more general situation than (8) can be 
modeled [10] by the noise with the power spectrum

$$
\Phi(\omega)=\frac{4 \Gamma \tilde{D}}{\left(\omega^{2}-\omega_{0}^{2}\right)^{2}+4 \Gamma^{2} \omega^{2}},
$$

where $\Gamma$ is a measure of the bandwidth, and $\omega_{0}$ the frequency of the noise. This can be thought of as the power spectrum of the system,

$$
\begin{gathered}
\ddot{f}+2 \Gamma \dot{f}+\omega_{0}^{2} f=\xi(t) \\
<\xi(t) \xi(s)>=4 \Gamma \tilde{D} \delta(t-s) .
\end{gathered}
$$

In this case $F(0)=\omega_{0}^{4} D / 4 \Gamma \tilde{D}$, and $F^{\prime \prime}(0)=\left(2 \Gamma^{2}-\omega_{0}^{2}\right) D / \Gamma \tilde{D}$. Obviously, $F^{\prime \prime}(0)>0$ when $\omega_{0}^{2}<2 \Gamma^{2}$ and thus the maximum of the power spectrum (8) is at $\omega=0$. In this case the direction of the current for $\Gamma \gg t_{r}^{-1}$ is the same as for exponentially correlated noise and the analysis given above directly applies.

A completely different situation occurs if $\omega_{0}^{2}>2 \Gamma^{2}$ and the power spectrum (8) has a minimum at $\omega=0$. In this case $F^{\prime \prime}(0)<0$. It follows from (5) that the direction of the current is opposite to that arising for exponentially correlated noise. In contrast to the case $F^{\prime \prime}(0)>0$ considered above, in the present case increase in $\left|F^{\prime \prime}(0)\right|$ does not give rise to saturation and then to decrease in $j$ : the current is increasing exponentially with the increasing $\left|F^{\prime \prime}(0)\right|$ where the approximation (3) is applicable. The current reversals have been found recently in numerical experiments, and in certain specific exactly solvable cases, by Doering and Horsthemke[2]. No plausible physical mechanism has been suggested as an explanation in [2]. Not only does the present analysis give an analytic criterion for the current reversal, $\Phi^{\prime \prime}(0) \propto-F^{\prime \prime}(0)>0$, but it also suggests a direct physical interpretation.

The correction to the activation energy of a transition $R$ in the case of "weakly colored" noise is due to the fact that it is not only the total work the noise does on the system on the way from the potential minimum to the barrier top (along the optimal path of the escape) that counts, as in the case of white noise, but the characteristic strength and duration of the pulse that gives rise to the escape. The parameters $\gamma_{ \pm}$just characterize the ratio of the squared value of the force to the duration of the pulse (cf. [6]). The shorter the pulse the higher are the frequencies of the noise components involved. If the power spectrum of the noise $\Phi(\omega)$ decreases with $\omega\left(F^{\prime \prime}(0)>0\right)$ then 
the higher-frequency noise components are weaker on the average, and the escape probability decreases with the decreasing duration of the pulse. For $\Phi(\omega)$ increasing with $\omega\left(F^{\prime \prime}(0)<0\right)$ the result is exactly opposite. The characteristic duration of the pulse is determined by the ratio of the distance between the potential minimum and the barrier top to the characteristic velocity $U^{\prime}$, and for a given height of the potential barrier it scales as $\left(U^{\prime}\right)^{-2}$.

Suppose we have a potential more steep to the left from the minimum, as shown in Fig.1. It is clear from (3) that in this case $\gamma_{-}>\gamma_{+}$. The pulse which gives rise to the escape over the left barrier is shorter, and the characteristic frequencies involved are higher. Therefore, for $F^{\prime \prime}(0)>0$ (as in the case of exponentially correlated noise), the escape over the left barrier is less likely to happen than that over the right one, and the current flows to the right, whereas for $F^{\prime \prime}(0)<0$ it flows to the left.

The behavior of the system when driven by slow noise $\left(t_{c}>>t_{r}\right)$ is also of interest. The activation energies in this limit can also be calculated via the method of Ref. [6], and are given by $R_{ \pm}=D\left[U_{m, \pm}^{\prime}\right]^{2} / 2 \phi(0)$ where $\left|U_{m, \pm}^{\prime}\right|$ are the maximum values of $\left|U^{\prime}\right|$ on the intervals $\left(x_{0}, x_{ \pm}\right)$. We note here in passing that there are nonanalytic $\left[\left(t_{r} / t_{c}\right)^{2 / 3}\right]$ corrections to $R_{ \pm}$[6, 9]. The current which arises in the case of slow noise is then

$$
j=\lambda\left[C_{+} \exp \left(-\left[U_{m,+}^{\prime}\right]^{2} / 2 \phi(0)\right)-C_{-} \exp \left(-\left[U_{m,-}^{\prime}\right]^{2} / 2 \phi(0)\right)\right] .
$$

$\left(C_{ \pm}\right.$are the constants that allow for the prefactors in the expressions for $W_{ \pm}$; obviously, $\phi(0)$ is just the mean-square value of the noise). What is happening here also has a clear physical interpretation (cf. \&]). Since the noise has an extremely long correlation time, in the range where $U^{\prime \prime}(x)>0$ the particle simply follows the force adiabatically according to $U^{\prime}(x(t))=$ $f(t)$. The fluctuation large enough to allow the particle to escape over a barrier is just the one that overcomes the restoring force $-U^{\prime}(x)$ for all $x$, and the probability of such a fluctuation is just $W \propto \exp (-R / D)$. The current in this case is always in the positive direction for $\left[U_{m,-}^{\prime}\right]^{2}>\left[U_{m,+}^{\prime}\right]^{2}$. There is again a saturation effect, and the current is maximized for $\phi^{-1}(0) \approx$ $4 D \ln \left(\left|C_{-} U_{m,-}^{\prime} / C_{+} U_{m,+}^{\prime}\right|\right) /\left(\left[U_{m,-}^{\prime}\right]^{2}-\left[U_{m,+}^{\prime}\right]^{2}\right)$.

We emphasize that the onset of current in the system considered is the result of it being away from thermal equilibrium. This means that dissipation and fluctuations are not interrelated via fluctuation-dissipation theorem. In the particular case considered dissipation was not retarded (the friction force 
is determined by the instantaneous value of the coordinate), and therefore the effect arises when the power spectrum of the noise displays dispersion (for Gaussian noise). A current can also arise in the situation of a white-noise driven system where dissipation is retarded.

Lastly we remark that the system can be expected to exhibit behavior similar to stochastic resonance as the noise strength $D$ is varied, with $j$ exhibiting a maximum for some noise strength. Obviously for very small $D$ the current is an increasing function of $D$. As the noise strength is increased the fluctuations will begin to wash out the effects due to the shape of the potential, leading to a decrease of the current for large $D$.

\section{References}

[1] M. Magnasco, Phys. Rev. Lett. 71, 1477 (1993).

[2] C. Doering, W. Horsthemke, and J. Riordan, Phys. Rev. Lett. (to appear).

[3] This term was apparently introduced by M. Magnasco.

[4] C. Peskin, G. Odell and G. Oster, Biophys. J. 65, 316 (1993).

[5] K. E. Drexler, Nanosystems: Molecular Machinery, Manufacturing, and Computation. Wiley-Interscience, New York, 1992.

[6] M. I. Dykman, Phys. Rev. A 42, 2020 (1990).

[7] J.F. Luciani and A.D. Verga, Europhys. Lett. 4, 255 (1987); M.M. Klosek-Dygas, B.J. Matkowsky, and Z. Schuss, SIAM J. Appl. Math 48, 425 (1988).

[8] G. Tsironis and P. Grigolini, Phys. Rev. Lett. 61, 7 (1988); A.J. Bray and A.J. McKane, Phys. Rev. Lett. 62, 493 (1989); A.J. McKane, Phys. Rev. A 40, 4050 (1989).

[9] A.J. Bray, A.J. McKane, and T.J. Newman, Phys. Rev. A 41, 657 (1990); R. Mannella, V. Palleschi, and P. Grigolini, Phy. Rev. A 42, 5946 (1990). 
Figure 1: Typical ratchet potential $U(x)$.

Figure 2: The sawtooth potential which is the "optimal" limit of $U(x)$ which gives rise to the greatest current.

[10] M.I.Dykman and K. Lindenberg, in Some Problems in Statistical Physics, ed. by G.H. Weiss (SIAM, Philadelphia, 1993). 
Figure 3: Current vs. $\tau$ for varying potential shape showing that the current is maximized for a specific $\tau$. 